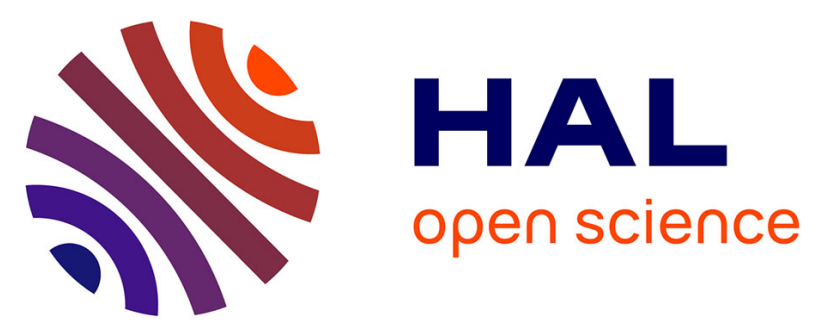

\title{
Minimizing Blocking Probability for the Multicast Routing and Wavelength Assignment Problem in WDM Networks: Exact Solutions and Heuristic Algorithms
}

\author{
Dinh Danh Le, Feng Zhou, Miklós Molnár
}

\section{- To cite this version:}

Dinh Danh Le, Feng Zhou, Miklós Molnár. Minimizing Blocking Probability for the Multicast Routing and Wavelength Assignment Problem in WDM Networks: Exact Solutions and Heuristic Algorithms. Journal of Optical Communications and Networking, 2015, 7 (1), pp.36-48. 10.1364/JOCN.7.000036 . lirmm-01349065

\section{HAL Id: lirmm-01349065 \\ https://hal-lirmm.ccsd.cnrs.fr/lirmm-01349065}

Submitted on 16 Apr 2021

HAL is a multi-disciplinary open access archive for the deposit and dissemination of scientific research documents, whether they are published or not. The documents may come from teaching and research institutions in France or abroad, or from public or private research centers.
L'archive ouverte pluridisciplinaire HAL, est destinée au dépôt et à la diffusion de documents scientifiques de niveau recherche, publiés ou non, émanant des établissements d'enseignement et de recherche français ou étrangers, des laboratoires publics ou privés. 


\title{
Minimizing Blocking Probability for MCRWA problem in WDM Networks: Exact Solutions and Heuristic Algorithms
}

\author{
Dinh Danh Le, Fen Zhou, and Miklós Molnár
}

\begin{abstract}
Given a sparse splitting WDM network, a set of available wavelengths, we investigate the problem of provisioning a set of multicast requests simultaneously with the objective of minimizing the blocking probability. Two blocking policies are taken into account: full blocking probability and partial blocking probability. As the problem is NP-hard, we propose an Integer Linear Programming (ILP) formulation with two variants (each for a blocking policy) to search for the optimal solution and several efficient adaptive heuristic algorithms to compute approximated solutions. Specially, instead of using light-trees, both ILP and heuristics use light-hierarchy, a recently optimal route under sparse splitting configurations. Extensive simulations reveal that our adaptive algorithms are able to compute nearoptimal solution and they outperform static approaches under both blocking probability policies. The results also show that it is more advantageous to provision multiple multicast communications with light-hierarchies, since they are able to accommodate more requests and destinations compared with the light-tree solutions.
\end{abstract}

Index Terms-WDM Networks, Multicast Routing and Wavelength Assignment (MCRWA), Sparse Splitting, Light-hierarchy, Full/Partial Blocking Probability, Adaptive/Static Routing, Integer Linear Programming (ILP)

\section{INTRODUCTION}

Wavelength division multiplexing (WDM) is becoming a perfect core network infrastructure for communications with huge bandwidth capacity, data transparency and low latency. According to the Cisco traffic forecast report [1], the average global Internet traffic will increase threefold over the next five years between 2012 and 2017. Applications accounting for this growing mainly include video sharing between data centers, Internet Protocol Television (IPTV), Voice over Internet Protocol (VoIP), video conferences, etc., in which both bandwidth and quality of services (QoS) requirements are crucial regarding the members of multicast groups. Consequently, all-optical multicasting techniques are indispensable for accommodating aforementioned bandwidth-intensive and QoS-sensitive applications.

To support WDM multicasting efficiently, the networks should be equipped with multicast capable cross-connects (MC-OXCs) that are capable of splitting an incoming signal to

Manuscript received June 26, 2014. This work is supported in part by VIED, Vietnam.

Dinh Danh Le, and Miklós Molnár are with the LIRMM lab at the University of Montpellier 2, France. Emails: \{dinhdanh.le, miklos.molnar\}@lirmm.fr.

Fen Zhou is with CERI-LIA (Computer Science Laboratory) at the University of Avignon, France. Email: fen.zhou@univ-avignon.fr. multiple outgoing ports. However, they are costly in both fabrication and power consumption. Thus, it is common in current optical networks that not all but a subset of nodes are multicast capable, giving rise to the sparse splitting configuration [2]. The fact that a small percentage of MC-OCXs mixed with multicast incapable cross-connects (MI-OXCs) may suffice to support optical multicasting [3].

Traditionally, light-tree [4] was assumed to be a costeffective route with the support of MC-OXCs at the branching nodes. Recently, a new multicast structure called lighthierarchy was proposed in [5]. A light-hierarchy allows an MI-OXC to be crossed several times by using different inputoutput link pairs, and hence relaxes the constraint of tree structure required by the routing.

The routing and wavelength assignment (RWA) problem in all-optical networks consists in finding a route and assigning a wavelength for each connection request such that two routes cannot be assigned with the same wavelength if they share a common link. This constraint is known as the distinct wavelength constraint or wavelength clash constraint [6]. Moreover, in the absence of wavelength converters, another constraint namely wavelength continuity constraint must be satisfied: the same wavelength should be retained in all the links of the computed route. This problem was earlier posed just for unicast connections with the name Lightpath Establishment problem [7]. It was then extended to the case of multicast traffic for the names MCRWA [8] or MC-RWA [9]. For the sparse splitting networks, the MCRWA problem is subject to the sparse splitting constraint, i.e., only MC-OXCs can be branching nodes in the computed routes (e.g., lighttrees) while MI-OXCs cannot.

The (MC)RWA problem can be either static or dynamic, depending on static or dynamic traffic patterns, respectively [10]. Among the two, the static (MC)RWA problem is more challenging since it has to deal with multiple requests simultaneously. The static routing and wavelength assignment is called off-line RWA since all the requests are known beforehand. The objective is mainly to minimize the resources (i.e., the number of wavelengths used, and/or the total link cost) provided that wavelength availability is sufficient to route all the requests; or to maximize the total number of requests provisioned, or equivalently, to minimize the blocking probability in the case of limited wavelengths.

Regarding blocking probability, two policies can be used: full destination blocking probability $(F B)$ and partial destination blocking probability (PB) [8], [9]. Accordingly, under 
FB policy, a multicast request is established if the source can reach to all the destinations. The appropriate metric to evaluate the solutions is session blocking probability (SBP). It is suitable for the applications that require all the multicast members receiving the data to take place, e.g., teleconferencing, distributed databases and distributed computing. On the other hand, in other applications such as video-on-demand, it may not be obligatory to reach all destinations at the same time. Rather, if some of the destinations are not reachable through the selected route, the connection is set-up between the source and the reachable destinations. This is the case where PB policy is supposed to use. Under PB policy, the metric of number of multicast sessions accepted (or SBP) is not suitable to evaluate the solutions. Instead, it is more appropriate to evaluate the maximum number of destinations served for all multicast sessions, i.e., the destination blocking probability (DBP).

In this paper, we deal with the static MCRWA problem in sparse splitting WDM networks (abbreviated as MCRWA-SS) where costly wavelength conversion is not available. We aim at minimizing the blocking probability of multicast demands for a given number of wavelengths. Two blocking models (FB and $\mathrm{PB}$ ) with the corresponding metrics (SBP and DBP) are taken into account. To this end, we propose an Integer Linear Programming (ILP) formulation with two variants (each for a blocking policy) to search for the optimal solution. Since the ILPs are not able to run with large instances, we propose several scalable adaptive MCRWA strategies that integrate the routing and wavelength assignment tasks in one step. Specially, both exact solutions and heuristics compute lighthierarchies for each request instead of light-trees. Extensive simulations carried out on realistic optical networks reveal that our adaptive algorithms are able to compute near-optimal solution and they outperform the static (including fixed and fixed-alternate) MCRWA approaches. The results also show that light-hierarchies can help to reduce blocking probability better than light-trees.

The rest of the paper is organized as follows. The related work is reviewed in Section II. The problem of MCRWA is presented in Section III. Section IV gives a brief definition of hierarchies in graphs and light-hierarchy structure. The two ILP formulations for computing light-hierarchies are presented in Section V. In Section VI, after introducing the layered graph model, we present several adaptive heuristic algorithms. Numerical results are shown in Section VII to evaluate the performances of the proposed algorithms. The paper is concluded in Section VIII.

\section{RELATED WORK AND MOTIVATION}

The RWA problem was proved to be NP-hard [7]. The MCRWA problem is therefore NP-hard since it contains the RWA problem as a special case [3]. Solving the static (MC)RWA problem can be done either by integrating the routing (R) and wavelength assignment (WA) in one step (coupled approach), or by separating them into two separated steps (decoupled approach).

In the coupled approach, the routes are decided and then assigned wavelengths depending on the network status. Since the network state changes dynamically during the route computation, this approach belongs to dynamic (or adaptive) RWA. Typical work of this approach include [6], [11]-[13]. In the decoupled (MC)RWA approach, a predetermined set of routes are computed in the physical topology as possible candidate routes for each connection request. Therefore, the routes cannot be changed during the wavelength assignment and this approach belongs to static RWA. The predetermined route set contains either a single route (in fixed routing) or multiple ones (in fixed-alternate routing) [14]. When a request is considered in the RWA process, these fixed routes are examined one by one until one is accepted. The request is blocked if all the routes are attempted without success. Most of the previous work employed the decoupled approach: [14]-[19].

Among the static (MC)RWA problems, the one for unicast traffic was extensively studied in the literature. Typical work can be found in [6], [18], [20], [21]. In [18], several static RWA strategies resulted from possible combinations of static routing and First-Fit wavelength assignment [14] are proposed to minimize the blocking probability. The authors in [6] studied the routing and wavelength assignment of static lightpath requests with the objective to minimize the number of wavelengths used. They proposed several adaptive heuristic algorithms by applying classical bin-packing algorithms. By taking both the ideas of bin-packing method and edge-disjoint paths, the authors in [21] proposed an GA-based algorithm to solve the static RWA problem. This algorithm outperforms the bin-packing based algorithms with a high computational time complexity. In [20], two static and three dynamic algorithms to reduce the blocking probability were also proposed. These algorithms resulted from the combination of fixed and fixedalternate routes with First-Fit, Random, Most-Used and LeastUsed [14] wavelength assignment schemes.

Besides, lots of published work in the literature [2], [5], [22]-[25] search for better solutions for the MCRWA problem under sparse splitting networks. However, they just focus on one multicast request, which do not fully reflect the nature of the MCRWA problem.

Few studies, however, tackled static MCRWA for multiple multicast requests [8], [9], [13], [19]. The static MCRWA problem was first studied in [8] and [9] targeting to the objective of minimizing the blocking probability. The work [19] focused on minimizing the number of wavelength converters (WCs) that are required to support the entire group of static multicast requests. However, these studies assumed to work on the networks wherein nodes are equipped with full splitting capability and/or wavelength converters, which does not closely reflect the currently sparse splitting network configurations. In [13], the authors studied the MCRWA problem under sparse splitting and sparse wavelength conversion networks. They proposed several heuristic algorithms based on "quasi-Prim" and "quasi-Kruskal" heuristics. Although the multi $\lambda$-light-trees are used instead of light-trees, they do not make use of possible arcs as the light-hierarchies.

Recently, the multicast-related resource allocation in spectrum elastic optical networks (EONs) has been received lots of attention in the literature in order to better utilize fiber's bandwidth. The typical works [26], [27] tried to minimize the 
blocking probability of multicast requests in EONs. However, only full blocking probability policy was taken into account, and the multicasting in EONs is not considered in our current work.

From the above analysis, there are two issues making the static MCRWA-SS problem challenging: multiple requests present together, and the sparse splitting constraint without wavelength conversion. This challenge builds up our motivation to tackle the problem.

\section{MCRWA-SS PROBLEM DEFINITION}

For simplicity, we assume that the MC-OXCs are realized by nonblocking Splitter-and-Delivery ( $\mathrm{SaD}$ ) switches [28] that can split an incoming light signal to as many outgoing ports as possible. Besides, we suppose that every MI-OXC supports Tap-and-Continue (TaC) [29]. A TaC-OXC can tap a small portion of power to the local station (when passing by a destination) and forward the remaining to one of the outgoing ports. Finally, no wavelength converter is available in the network.

A WDM network topology is represented by a digraph $G=$ $(V, E)$ where $V$ is a set of OXCs. Under sparse splitting, $V=M C \cup M I$, in which $M C$ is the set of MC-OXCs, $M I$ is the set of MI-OXCs. A link between two adjacent OXCs $u$ and $v$ consists of two optical fibers $(u, v)$ and $(v, u)$ in charge of the communications for two opposite directions. All the optical fibers constitute the arc set $E$, in which each arc $(u, v) \in E$ is associated with a cost $c_{u v}$. We suppose that all optical fibers support the same set of wavelengths $W=$ $\left\{\lambda_{1}, \lambda_{2}, \ldots, \lambda_{|W|}\right\}$. A set of multicast requests $R$ are known beforehand, $R=\left\{r_{i}, i \in \mathcal{I}\right\}$, in which $r_{i}=\left(s_{i}, D_{i}\right): s_{i} \in$ $V, D_{i} \subseteq V \backslash\left\{s_{i}\right\}, \mathcal{I}=\{1,2, . .,|R|\}$. For each request, a set of light-structures $L S$ (e.g., light-trees or light-hierarchies) is computed to delivery the multicast message from the source $s_{i}$ to the set of destinations $D_{i}$ while satisfying all the three constraints mentioned in Section I.

To evaluate the solutions, we define two quantitative metrics: session blocking probability (SBP) and destination blocking probability (DBP). The SBP corresponds to the full blocking policy, and the DBP corresponds to the partial blocking policy. Let $B_{i}, B_{i}^{d}$ be two set of binary variables such that: $B_{i}=1$ if request $r_{i}$ is accepted, 0 otherwise; and $B_{i}^{d}=1$ if destination $d$ of request $r_{i}$ is accepted, 0 otherwise. SBP and DBP are defined as follows:

$$
\begin{aligned}
& S B P=1-\frac{\sum_{i \in \mathcal{I}} B_{i}}{|R|} \\
& D B P=1-\frac{\sum_{i \in \mathcal{I}} \sum_{d \in D_{i}} B_{i}^{d}}{\sum_{i \in \mathcal{I}}\left|D_{i}\right|}
\end{aligned}
$$

Formally, the MCRWA-SS problem can be defined as follows.

- Input: An WDM network $G=(V, E)$, a set of wavelengths $W$ and a set of multicast requests $R=\left\{r_{i}, i \in \mathcal{I}\right\}$

- Output: A set of light-structures satisfying the three constraints
- Objective: Minimize $S B P$ under FB policy or minimize $D B P$ under PB policy

\section{LIGHT-HIERARCHY TO SOLVE THE MCRWA PROBLEM}

In all-optical WDM networks, light-trees have been proposed for MCRWA problem. However, the tree structure restricts the network links to be passed no more than once, even though there is another (opposite) direction on the same link available to use. (Recall that in our study, we suppose that there are two (opposite) directed fibers in order to provide both directions in each link.) To make use of the possible directions in each link, the concept hierarchy is proposed in [30] to replace the traditional solutions (e.g., path, tree, etc.).

\section{A. Hierarchies in graphs}

Hierarchy is a graph related structure obtained by a homomorphism of a tree in a graph which is defined as follows. Let $Q=(W, F)$ and $G=(V, E)$ be two graphs. A hierarchy $H$ is obtained by an application $h: W \rightarrow V$ mapping a vertex in $W$ to each vertex in $V$ under two conditions: (1) $Q$ is a tree and (2) $h$ is a homomorphism. Recall that $h$ is a homomorphism if the mapping preserves the adjacency: $(u, v) \in F \Rightarrow(h(u), h(v)) \in E$. Fig. 1 gives an example of a mapping $h$ from a tree $Q$ to a graph $G$ for a hierarchy $H$. Each vertex of tree $Q$ is associated with a unique vertex of $G$. In the reverse direction, some vertices of $G$ are mapped from several vertices in $Q$ (vertices $d$ and $e$ ). We label the vertex (vertices) in $Q$ associated to the same vertex $v$ in $G$ as $v^{1}, v^{2}, \ldots, v^{k}$. Especially, when the mapping $h$ is injective, the hierarchy corresponds to a tree. The reader is encouraged to refer to [30] for a full definition with detail properties and possible applications of hierarchies.

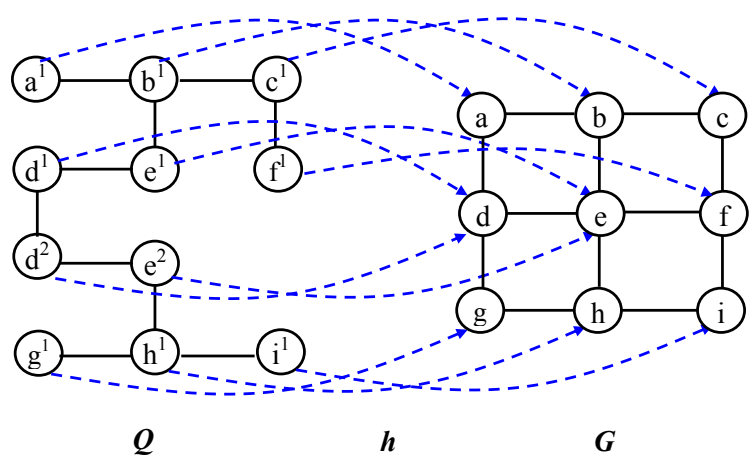

(a)

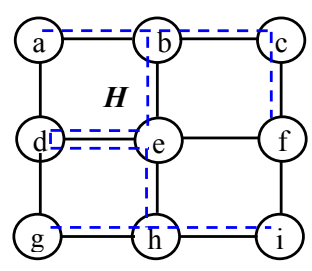

(b)

Fig. 1: Mapping of vertices for a hierarchy 


\section{B. Light-hierarchy versus Light-tree}

A light-hierarchy is a directed partial spanning hierarchy realized by using a single wavelength respecting the optical constraints. Equivalently, a light-hierarchy is a hierarchy that has no duplicated arc but is free of repetition of nodes. Thus, light-hierarchy is a general case of light-tree.

An example showing the advantages of light-hierarchy over light-tree can be seen in Fig. 2. The network topology is in solid line; all the nodes are MI-OXCs except node 1, an arrival multicast request $\left(s,\left\{d_{1}, d_{2}\right\}\right)$. The optimal light-tree solution is shown in the dash-dotted line in Fig. 2(a)) with the total cost of 7 (supposed that all links have identical cost). However, a light-hierarchy can be found by using different ingress-ogress fibers crossing node 3 as shown in Fig. 2(b) with the total cost of 6 . The solution can still be improved by another lighthierarchy that utilizes the links in both directions as shown in Fig. 2(c) with the total cost of 5. Obviously, the light-hierarchy solution outperforms the light-tree solution.

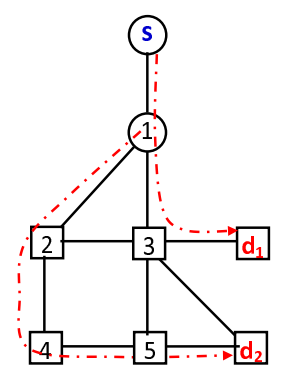

(a) A light-tree

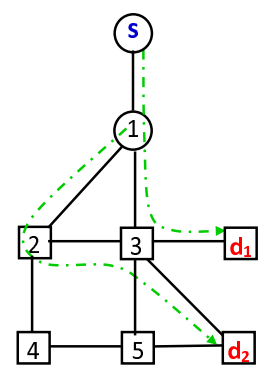

(b) light-hierarchy

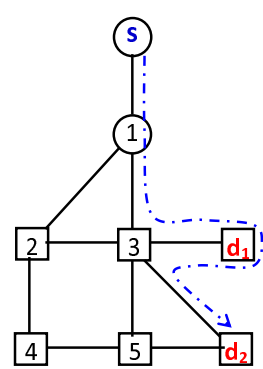

(c) light-hierarchy 2
Fig. 2: Light-hierarchy solution versus light-tree solution

\section{LIGHT-HIERARCHY BASED ILP FORMULATIONS WITH Full BLOCKING AND PARTIAL BlockING POLICY}

With light-hierarchy structure, the sparse splitting constraint could be relaxed to some extent. Consequently, more destinations may be served in one light-hierarchy. This is why the light-hierarchy structure can be the optimal solution. In this section, the integer linear programming (ILP) method is applied to search for the optimal light-hierarchy solution under each of aforementioned blocking probability (FB and PB) policies. In general, the two ILP formulations are similar to each other. They differ from one another in the objective function and some related constraints.

To formulate, some notations mentioned in Section III are reused, including $G, V, E, W, R$. Besides, several additional notations are predefined in Table I and four ILP variable vectors $L_{u v}^{i \lambda} \in\{0,1\}^{|R| \times|W| \times|E|}, \quad F_{u v}^{i \lambda} \in \mathbb{N}^{|R| \times|W| \times|V|}$, $B_{i} \in\{0,1\}^{|R|}$, and $B_{i}^{d} \in\{0,1\}^{|R| \times\left|D_{i}\right|}$ as in Table II.
TABLE I: Network Parameters

\begin{tabular}{|ll|}
\hline \hline$\lambda$ & $:$ A wavelength, $\lambda \in W$. \\
$N^{+}(v)$ & $:$ Extremities of the outgoing arcs from OXC $v$. \\
$N^{-}(v)$ & $:$ Extremities of the incoming arcs to OXC $v$. \\
$D e g$ & $:$ The maximum nodal degree in $G$. \\
$(u, v)$ & $:$ The arc from OXC $u$ to $v$. \\
$c_{u v}$ & $:$ The cost of the arc from OXC $u$ to $v$. \\
$\Delta$ & $:$ An integer big enough such that $\Delta>|W| \times \sum_{(u, v) \in E} c_{u v}$. \\
$M C$ & $:$ The set of MC-OXCs in $G$. \\
$M I$ & $:$ The set of MI-OXCs in $G$. \\
$i \in \mathcal{I}$ & $:$ The index $i$ The index set $\mathcal{I}$ of $R$. \\
$f_{i}$ & $:$ The importance of multicast request $r_{i}$. \\
\hline \hline
\end{tabular}

\section{TABLE II: ILP Variables}

\begin{tabular}{|ll|}
\hline \hline$L_{u v}^{i \lambda} \in\{0,1\}$ & $:$ Equals 1 if multicast request $r_{i}$ uses \\
& wavelength $\lambda$ on link $(u, v)$, \\
& equals 0 otherwise. \\
$F_{u v}^{i \lambda} \in\left[D_{i}\right] \quad$ & Commodity flow. Denotes the number of \\
& destinations in request $r_{i}$ \\
& receiving a flow from $s_{i}$ via arc $(u, v)$ on $\lambda$. \\
$B_{i} \in\{0,1\}$ & $:$ Equals 1 if request $r_{i}$ is accepted, 0 otherwise. \\
$B_{i}^{d} \in\{0,1\}$ & $:$ Equals 1 if destination $d$ of request $r_{i}$ is accepted, 0 \\
& otherwise. \\
\hline
\end{tabular}

OXCs in the request $r_{i}$ receiving a flow from $s_{i}$ via arc $(u, v)$ on $\lambda$, and binary variable $B_{i}$ decides whether the request $r_{i}$ is accepted. As we suppose that the wavelength availability is limited, the original objective of our problem is to minimize the session blocking probability (SBP), or equivalently to maximize the number of accepted requests. To provide a flexible solution, we introduce a variable $f_{i}$ for the importance of the request $r_{i}$, and objective is now to maximize the overall importance of all accepted multicast requests, i.e., $\sum_{i \in \mathcal{I}} f_{i} \cdot B_{i}$. Secondly, we also try to minimize wavelength channel cost. To this end, a big enough integer $\Delta$ should be introduced such that the contribution of $\Delta \times \sum_{i \in \mathcal{I}} f_{i} \cdot B_{i}$ in the overall objective function should be much bigger than the wavelength channel cost times $|W|$, i.e. $\Delta>|W| \times \sum_{(u, v) \in E} c_{u v}$.

Hence the general objective function can be expressed as follows:

$$
\text { Maximize }: \Delta \cdot \sum_{i \in \mathcal{I}} f_{i} \cdot B_{i}-\sum_{i \in \mathcal{I}} \sum_{\lambda \in W} \sum_{(u, v) \in E} c_{u v} \cdot L_{u v}^{i \lambda}
$$

\section{A. ILP with Full Blocking Policy (ILP-FB)}

For ease of presentation, let $L H^{i \lambda}$ be a light-hierarchy on wavelength $\lambda$, which is used for the request $r_{i}$. The binary variable $L_{u v}^{i \lambda}$ denotes whether the arc $(u, v)$ is used by $L H^{i \lambda}$, integer variable $F_{u v}^{i \lambda}$ represents the number of destination

subject to the light-hierarchy constrains (2)-(8) and the connectivity constraints (9)-(15). In the following, we use $\forall i, \forall d$, $\forall \lambda$, and $\forall(u, v)$ to imply $\forall i \in \mathcal{I}, \forall d \in D_{i}, \forall \lambda \in W$, and $\forall(u, v) \in E$ respectively. 
$\begin{array}{ll}\sum_{v \in N^{-}\left(s_{i}\right)} L_{v s_{i}}^{i \lambda}=0, & \forall i, \forall \lambda \\ \sum_{u \in N^{-}(v)} L_{u v}^{i \lambda} \leq B_{i}, & \forall i, \forall \lambda, \forall v \in M C \backslash\left\{s_{i}\right\} \\ \sum_{u \in N^{+}(v)} L_{v u}^{i \lambda} \leq \sum_{u \in N^{-}(v)} L_{u v}^{i \lambda} \cdot D e g, \quad \forall i, \forall \lambda, \forall v \in M C \backslash\left\{s_{i}\right\}\end{array}$

$\sum_{u \in N^{+}(v)} L_{v u}^{i \lambda} \leq \sum_{u \in N^{-}(v)} L_{u v}^{i \lambda}, \quad \forall i, \forall \lambda, \forall v \in M I \backslash\left\{s_{i}\right\}$

$\sum_{u \in N^{+}(v)} L_{v u}^{i \lambda} \geq \sum_{u \in N^{-}(v)} L_{u v}^{i \lambda}, \quad \forall i, \forall \lambda, \forall v \notin D_{i}$

$\sum_{i \in \mathcal{I}} L_{v u}^{i \lambda} \leq 1$

$\forall \lambda, \forall(v, u)$

$L_{v u}^{i \lambda} \leq B_{i}$

$\forall i, \forall \lambda, \forall(v, u)$

For any $i \in \mathcal{I}$ and $\lambda \in W$, constraint (2) ensures that the light-hierarchies for a multicast request $r_{i}$ are rooted at the source node $s_{i}$. Constraint (3) guarantees that each MCOXC has at most one input arc in each light-hierarchy $L H^{i \lambda}$, while constraint (4) indicates that each MC-OXC is able to split the light signal. Constraints (4) and (5) make sure that no $\mathrm{OXC}$ in $V$ can be the root of $L H^{i \lambda}$ except the source node $s_{i}$. Constraint (5) together with constraint (6) indicates an intermediate MI-OXC may have multiple input links and multiple output links with the help of cross pair switching, but the number of input links must be equal to the number of output links. Constraint (6) determines that only the destinations in $D_{i}$ can be leaf nodes of a light-hierarchy $L H^{i \lambda}$. For all $i \in \mathcal{I}$, constraint (7) corresponds to the distinct wavelength constraint, which forbids two light-hierarchies to share a link using the same wavelength. Constraint (8) indicates that there is no link used for the blocked request.

With the above the light-hierarchy structure constraints, we can not guarantee the connectivity of the light-hierarchy. Thus, we propose a commodity flow method to impose supplementary constraints on variables $L_{u v}^{i \lambda}$ so that the connectivity of the resultant light-hierarchy could be guaranteed.

2) Connectivity Constraints: To establish a multicast request, several light-hierarchies may be required. And the same destination may be spanned by several light-hierarchies. However, a destination can only be served in one light-hierarchy to consume the light signal (i.e. receive the multicast messages), while it is spanned in the other light-hierarchies to uniquely forward the light signal to the successor node.

$$
\begin{array}{ll}
\sum_{\lambda \in W} \sum_{v \in N^{+}\left(s_{i}\right)} F_{s_{i} v}^{i \lambda}=\left|D_{i}\right| \cdot B_{i}, & \forall i \\
\sum_{\lambda \in W} \sum_{v \in N^{-}(d)} F_{v d}^{i \lambda}=\sum_{\lambda \in W} \sum_{v \in N^{+}(d)} F_{d v}^{i \lambda}+B_{i}, \forall i, \forall d \\
\sum_{v \in N^{-}(d)} F_{v d}^{i \lambda}-B_{i} \leq \sum_{v \in N^{+}(d)} F_{d v}^{i \lambda}, \forall i, \forall d, \forall \lambda \\
\sum_{v \in N^{-}(d)} F_{v d}^{i \lambda} \geq \sum_{v \in N^{+}(d)} F_{d v}^{i \lambda}, & \forall i, \forall d, \forall \lambda \\
\sum_{u \in N^{-}(v)} F_{u v}^{i \lambda}=\sum_{u^{\prime} \in N^{+}(v)} F_{v u^{\prime}}^{i \lambda}, & \forall i, \forall \lambda, \forall v \notin D_{i} \\
F_{u v}^{i \lambda} \geq L_{u v}^{i \lambda}, & \forall i, \forall(u, v), \forall \lambda \\
F_{u v}^{i \lambda} \leq\left|D_{i}\right| \times L_{u v}^{i \lambda}, & \forall i, \forall(u, v), \forall \lambda
\end{array}
$$

If multicast request $r_{i}$ is accepted, i.e., $B_{i}=1$, constraint (9) indicates that $s_{i}$ should generate $\left|D_{i}\right|$ commodity flow so that each destination of this multicast request can be served once. Constraints (10) and (11) ensure that each destination of request $r_{i}$ must consume one and only one commodity flow generated by $s_{i}$ in all the light-hierarchies built for this request. In other words, they guarantee each destination is reachable from the source $s_{i}$ in all light-hierarchies. Constraint (13) guarantees that the flow does not drop after passing a nonmember node. Relationship between $L_{u v}^{i \lambda}$ and $F_{u v}^{i \lambda}$ is expressed by constraints (14) and (15). They assure that a link should carry non-zero flow if it is used in a light-hierarchy, and the value of this flow should not beyond the total flow emitted by the source node.

\section{B. ILP with Partial Blocking Policy (ILP-PB)}

The ILP-PB is formulated based on the previous ILP-FB with some changes. With the partial blocking policy (PB), some destinations in a request may be rejected while the other destinations in the same request are served. Under the PB policy, the metric of number of multicast request accepted (or $S B P$ ) is not suitable for this scenario to evaluate the algorithm performance. Instead, it is more interesting to evaluate the maximum number of destinations served for all multicast requests (equivalently, to minimize the $D B P$ ). Thus, we use the ILP variable $B_{i}^{d}$ instead of $B_{i}$, and several constraints should be changed accordingly. Also, the link usage (resource) should be minimum. Accordingly, the new objective function can be expressed as:

$$
\text { Maximize : } \Delta \cdot \sum_{i \in \mathcal{I}} \sum_{d \in D_{i}} B_{i}^{d}-\sum_{i \in \mathcal{I}} \sum_{\lambda \in W} \sum_{(u, v) \in E} c_{u v} \cdot L_{u v}^{i \lambda}
$$

To adjust the previous ILP-FB model for the partial blocking policy, we replace constraints (9)-(11) by constraints (19)-(21).

Besides, the constraints (3) and (8) are changed as follows:

$$
\begin{array}{cr}
\sum_{u \in N^{-}(v)} L_{u v}^{i \lambda} \leq 1, & \forall i, \forall \lambda, \forall v \in M C \backslash\left\{s_{i}\right\} \\
L_{v u}^{i \lambda} \leq \sum_{d \in D_{i}} B_{i}^{d}, & \forall i, \forall \lambda, \forall(v, u)
\end{array}
$$


Constraint (17) makes sure the unique input link coming to any MC-OXCs. Constraint (18) guarantees that there is no link used for a request if all of its destinations are blocked.

Constraint (22) ensures that if $d$ of request $i$ is rejected, then $d$ will not have any input link reserved for request $i$ on any wavelength.

$$
\begin{aligned}
& \sum_{\lambda \in W} \sum_{v \in N^{+}\left(s_{i}\right)} F_{s_{i} v}^{i \lambda}=\sum_{d \in D_{i}} B_{i}^{d}, \quad \forall i \\
& \sum_{\lambda \in W} \sum_{v \in N^{-}(d)} F_{v d}^{i \lambda}=\sum_{\lambda \in W} \sum_{v \in N^{+}(d)} F_{d v}^{i \lambda}+B_{i}^{d}, \quad \forall i, \forall d \\
& \sum_{v \in N^{-}(d)} F_{v d}^{i \lambda}-B_{i}^{d} \leq \sum_{v \in N^{+}(d)} F_{d v}^{i \lambda}, \quad \forall i, \forall d, \forall \lambda \\
& L_{u d}^{i \lambda} \leq B_{i}^{d}, \quad \forall i, \forall d, \forall u \in N^{-}(d), \forall \lambda
\end{aligned}
$$

In short, ILP-PB is subject to light-hierarchy constraints: (2), (4)-(7) and (17)-(18), and connectivity constraints: (12)(15), and (19)-(22).

\section{Comparison of two ILP models}

In the proposed ILP-FB model, there are $|W| \times(2|R| \times|E|+$ 1) variables and $|R| \times|W| \times(3|V|+3|E|+|M C|)+(2+|R|) \times$ $|W| \times|E|+\sum_{i \in \mathcal{I}}\left|D_{i}\right|+|R|+|W|$ constraints. Regarding the ILP-PB model, there are $\sum_{i \in \mathcal{I}}\left|D_{i}\right|$ more variables and $|W| \times \sum_{i \in \mathcal{I}} \sum_{d \in D_{i}}\left|N^{-}(d)\right|$ more constraints.

The two ILP formulations above are run to search the exact solutions for the two problems corresponding to the two blocking policies. The results are used as the references to evaluate the proposed heuristics presented in the next sections.

\section{Heuristic Solutions}

In this section, we first present our implementation for existing static routing approaches, known as fixed and fixedalternate strategies. In order to design adaptive strategies, a layered graph model is introduced. We then describe the Arc Removal Light-Hierarchy (ARLH) algorithm which plays a core role in our proposed adaptive strategies presented afterwards.

\section{A. Existing Static Strategies}

For static strategies, we employ Member-Only (MO) algorithm [2] for routing and First-Fit scheme [14] for wavelength assignment. Note that due to the sparse splitting constraint, a single light-tree may not sufficient to accommodate a request. So a set of light-trees (i.e., a light-forest) may need to be computed. For the fixed strategy, the light-trees for each request are computed one-by-one for each request on the physical topology. Each light-tree is tried to assign an available wavelength (employing First-Fit) complying the distinct wavelength constraint until the wavelength pool is exhausted. Under full blocking (FB) policy, the requests having at least one unreachable destination are blocked. Thus, whenever a light-tree (say, for request $r$ ) is not accepted, the algorithm blocks $r$ by excluding it from the request set, freeing the wavelengths that have been assigned for the previous lighttrees for $r$, and continuing with the next request. On the other hand, if partial blocking (PB) policy is applied, all the lighttrees for $r$ are considered even though some of them may not be accepted. If request $r$ is not accepted, the reachable destinations are still adopted.

In fixed-alternate strategy, for a request we computed two arc-disjoint trees for each light-tree in the light-forest. If the first light-tree is not accepted, the alternate tree is tried. The request is blocked if none of the two light-trees is accepted. The two blocking policies are also applied in the same way as mentioned with fixed routing scheme above. In the simulations, we name MO-FIX for MO with fixed routing scheme and MO-ALT for MO with fixed-alternate routing scheme, respectively.

\section{B. Layered graph model}

In order to design coupled (adaptive) strategies for the considered MCRWA-SS problem, we modify the layered graph model that was first proposed in [11] to solve RWA with unicast traffic and then was used in [13] for the MCRWA problem. The proposed algorithms are operated on the layered graph instead of the physical topology. In this model, the physical topology is replicated into $|W|$ directed wavelength graphs $G_{\lambda}, \lambda=1,2, . .,|W|$ or layers, each layer corresponds to a distinct wavelength. Since we assume that no wavelength converter is available in the network, there is no interconnection between the layers. With the assumption that all the fibers have the same set of wavelengths, all the wavelength graphs (layers) are identical at the beginning. However, during the network operation, it is continuously changed to immediately reflect the current network state. Given a physical topology modelling a network with three wavelengths $\left(\lambda_{1}, \lambda_{2}, \lambda_{3}\right)$ as shown in Fig. 3(a), a constructed layered graph is shown in Fig. 3(b). Note that, the physical topology appears as an undirected graph, but its layered graph is a collection of bidirected graphs because we assume that each network link has two directed fibers (cf. Section III).
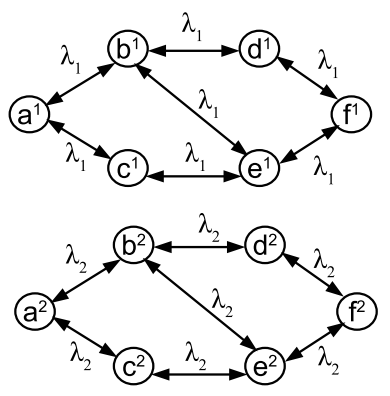

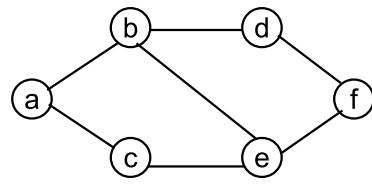

(a) A physical topology

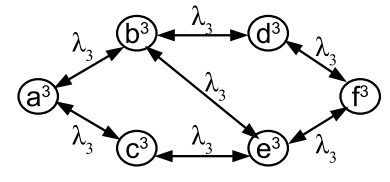

(b) A corresponding layered graph
Fig. 3: An example of a layered graph

Many interesting properties can be obtained from the layered graph model, which the physical topology model does 
not have, including [13]:

1) Solving MCRWA in one step. Wavelength assignment is accomplished when routing.

2) Low computational complexity and high performance. Any algorithm running on each wavelength graph generally takes no more computational time than running on physical topology graph. Moreover, since the wavelength assignment is done when routing, the time complexity of the WA subproblem is eliminated.

3) Immediately updating the latest network state. Whenever a wavelength is assigned, the corresponding arc is removed from the wavelength graph. Thus the layered graph keeps track of and reflects the updated status of the network.

\section{Arc Removal Light-Hierarchy Algorithm (ARLH)}

The Arc Removal Light-Hierarchy Algorithm is designed to compute a light-hierarchy for a multicast request $r$ on wavelength graph $G_{\lambda}$. As discussed in the previous part, an MI-OXC could be visited more than once in a lighthierarchy, while the arc already used in a sub light-hierarchy cannot be used any longer in the same light-hierarchy. As a result, the used arc in a sub light-hierarchy is useless for the expansion of the light-hierarchy to the remaining destinations of the considered request. From this point, an Arc Removal Light-hierarchy algorithm is designed to compute the light-hierarchies, such that the used arc is removed from the wavelength graph whenever it is used. ARLH employs the basic idea of the Minimum Path Heuristic [31].

Normally, ARLH computes one rooted light-hierarchy $(L H)$ at a time. However, it may produce no $L H$ due to the sparse splitting and/or lack of wavelengths on the wavelength graph. Also, the computed $L H$ may or may not cover all the destinations of the considered request. If the request is not entirely provisioned, the remaining of it will be reconsidered in the next runs. Initially, the $L H$ consists only the source of the multicast request (cf. Algorithm 1). ARLH then extends $L H$ by iteratively adding destinations one by one by the shortest paths. At each iteration, the algorithm finds the nearest destination $d$ from the $M C \_S E T^{1}$ of the current $L H$. Then the corresponding shortest path $S P(c, d)$ in wavelength graph $G_{\lambda}$ is added to $L H$ ( $c$ is the nearest node in $M C \_S E T$ to $d)$. Then the arcs in the shortest path $S P(c, d)$ are removed from the wavelength graph $G_{\lambda}$ and $M C \_S E T$ is updated. In the next iteration, the nearest destination and the shortest path are computed in the updated wavelength graph of $G_{\lambda}$. The algorithm terminates when there is no destination remaining, or the computed $L H$ cannot be extended. It is definitely true that the light-hierarchies computed respect the splitting constraint.

\section{Adaptive strategies for MCRWA-SS problem}

In what follows we describe several strategies for the MCRWA-SS problem. In these strategies the routing is performed by applying $A R L H$ algorithm on the layered graph to

\footnotetext{
${ }^{1} M C \_S E T$ includes the MC-OXCs and the leaf MI-OXCs in the current computed light-hierarchy.
}

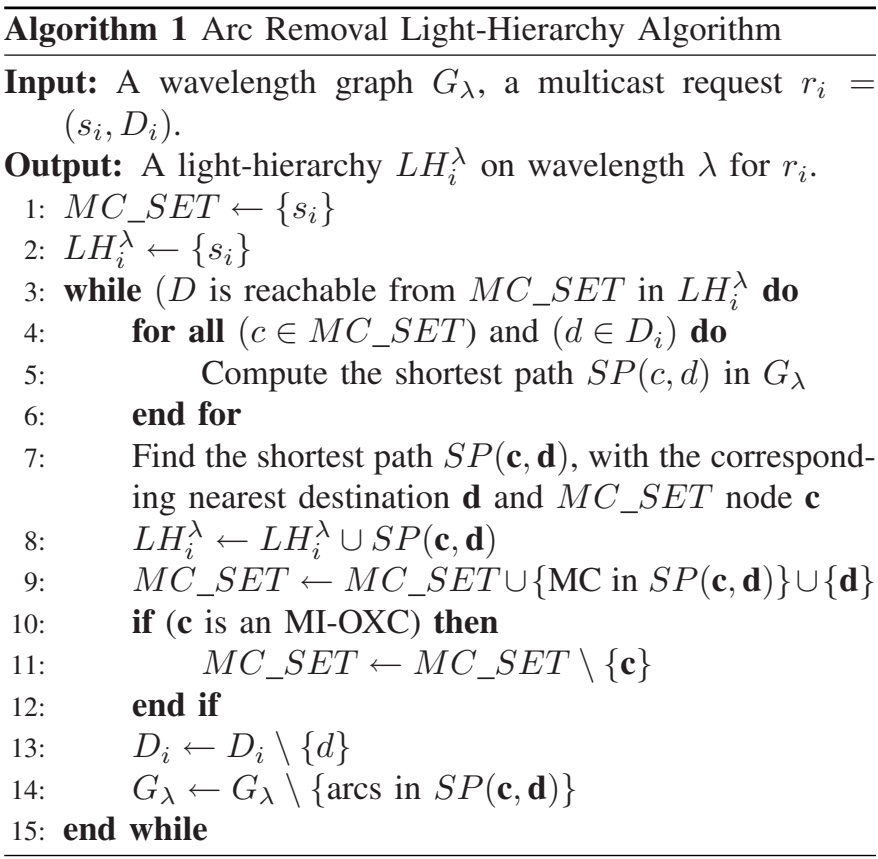

compute the light-hierarchies for the requests, then the lighthierarchies are assigned with the corresponding wavelengths straightforward. By this way, we integrate the routing and wavelength assignment tasks in one step.

1) Sequential Request (seqR): For each request $r_{i}$ to be considered (selected according to the indexed order $\left.r_{1}, r_{2} \ldots, r_{|R|}\right)$, compute the light-hierarchies employing ARLH on the layers (wavelength graphs) $\lambda_{1}, \lambda_{2}, . ., \lambda_{|W|}$ one by one (according to the indexed order). Whenever a light-hierarchy is computed in certain layer, it is directly assigned the corresponding wavelength. Then the affected layers are updated in such a way that the arcs used for wavelength assignment are removed from the wavelength graphs. Repeat the operations until all the requests are attempted. The pseudo code of SeqR algorithm is shown in Algorithm 2.

Note that we use both aforementioned blocking policies and apply them for all the proposed algorithms described below. Under FB policy, a request is considered to be accepted if all of its destinations are accepted. If not, the request is blocked, the algorithm frees the wavelengths (restore the status for the used arcs) that have been assigned for the light-hierarchies computed for it. In contrast, when PB policy is used, if the request is not totally accepted, the accepted destinations are still served.

This strategy is natural and relatively straightforward. It does not need the global information of all the requests. Thus it can be applied for the dynamic traffic case.

2) Smallest Request First (SRF): It is similar to seqR, for each iteration, except that it selects the smallest request (in terms of number of destinations or group size) to consider first. To this end, the requests are sorted beforehand according to the non-descending order of group size. After that it follows the same operations as $\operatorname{seq} R$ for all the requests considered. The idea of this strategy (choosing the smallest request first) based on the observation that more number of small items can be put into a bin with limited capacity than putting larger items 
first. $S R F$ can help to increase the total number of requests accepted, and hence can achieve lower the session blocking probability (SBP).

3) Largest Request First ( $L R F)$ : It is somewhat contrary to $S R F$, instead of provisioning the smallest request, it chooses the largest one first. By choosing the largest request sooner, more destinations might be adopted, hence $L R F$ can increase the total number of destinations served or increase the destination blocking probability (DBP). However, it can also increase the probability that small requests will easily be blocked, leading high SBP compared with $S R F$.

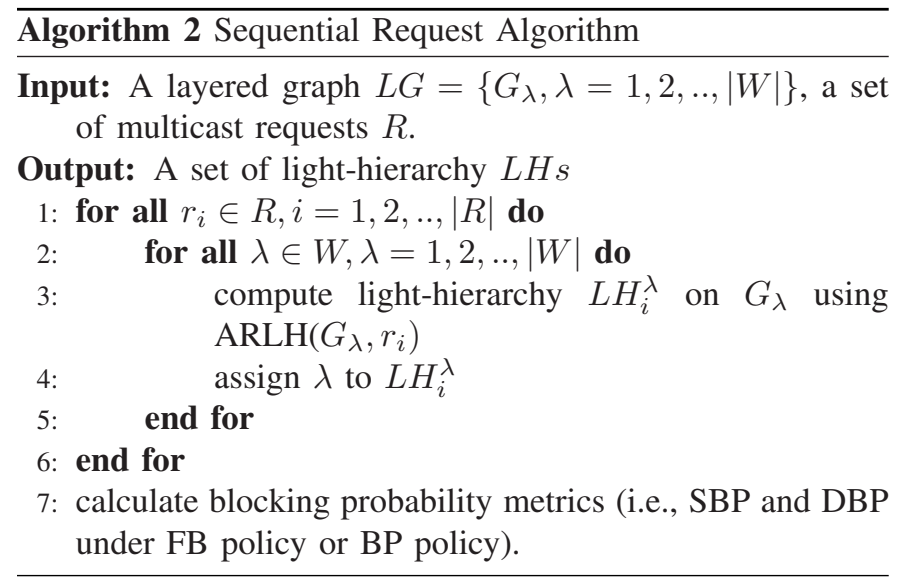

\section{E. Computational Complexity}

Theorem 1: Let $n$ be the number of destinations accepted for all the requests. The time complexity of the three adaptive algorithms (SeqR, SRF and $L R F)$ are $O(n \times(|V| \log |V|+|E|))$.

Proof: We analyse the time complexity of $\operatorname{SeqR}$ only, it is then deduced for the other two. Let us take a look in the Algorithm 2, the most expensive operation is in line 3 where ARLH is called. Since $W$ is limited, we suppose that $n_{i}$ is number of destinations of request $r_{i}$ that are reached $\left(0 \leq n_{i} \leq\left|D_{i}\right|\right)$. To find the nearest destination in $D_{i}$ from the $M C \_S E T$ in any wavelength graph $G_{\lambda}$, Dijkstra's algorithm should be used once. Because, the connector nodes in $M C \_S E T$ could be virtually gathered and viewed together as a virtual source (e.g., set their distances to the source as 0) and it is sufficient to find the nearest destination and the distances by constructing a single shortest path tree in $G_{\lambda}$ from the source to all the destinations. Since $n_{i}$ destinations are reached, $n_{i}$ steps are required. Thus the Dijkstra's algorithm should be used $n_{i}$ times during the construction of light-hierarchies for request $r_{i}$. The time complexity of Dijkstra's algorithm implemented by a Fibonacci heap is $O(|V| \log |V|+|E|)$ [32]. So, the time complexity of the proposed $S e q R$ algorithm is

$$
\sum_{i=1}^{|R|} n_{i} \times O(\text { Dijkstra })=n \times O(|V| \log |V|+|E|)
$$

Since $S R F$ and $L R F$ have one complement step of sorting the requests before calling $S e q R$, and this step needs much less time compared to the active operation (in line 3 ) of the Algorithm 2, we can state that all the three algorithms have the same complexity.

\section{Performance Evaluation}

We divide the simulations into two parts. The first part is to evaluate the proposed heuristics with the exact solutions obtained from corresponding ILP formulations with small instances. The second part presents the comparison among heuristics with larger realistic configurations.

\section{A. Heuristics versus ILP results}

Due to the exponential complexity of ILP formulations, only small instances can be possible. We use the 14-node NSF network (cf. Fig. 4(a)). To simulate with sparse splitting capacity, the ratio of MC-OXCs is set to $0 / 14$ and 3/14. Given $S$ MC-OXCs $(S=|M C|), 10$ multicast requests are randomly generated in a manner that the source and destinations are distributed randomly and uniformly in the networks. The number of wavelengths varies from 1 to 3 . The ILP were solved with CPLEX 12.5. Both ILPs and heuristics were run on a machine with Intel Core i3, $2.20 \mathrm{GHz}$ processor and 4GB of RAM. We ran ILPs for maximum of one-hour each run. If the solver cannot find the optimal solution within one hour, the quasi-optimal solution with the gap of $1 \%$ to the optimal is accepted. Otherwise, the instance is considered failed and our program directs the ILP to run on another instance. The simulation results are shown in Table III. Each value on the table is the average of 20 successful individual runs. For simplicity, we suppose that all requests have the same importance, i.e., $f_{i}=1, \forall i \in \mathcal{I}$.

TABLE III: Performance comparison of heuristics to ILP solutions.

\begin{tabular}{|c|c|c|c|c|c|c|c|c|c|}
\hline & & \multicolumn{2}{|c|}{$|W|=1$} & \multicolumn{2}{|c|}{$|W|=2$} & \multicolumn{2}{|c|}{$|W|=3$} & \multicolumn{2}{|c|}{ Avg Ratio } \\
\hline & Algos & $S=0$ & $S=3$ & $S=0$ & $S=3$ & $S=0$ & $S=3$ & $S=0$ & $S=3$ \\
\hline \multirow{6}{*}{$\frac{\sqrt{2}}{\frac{0}{6}}$} & MO-FIX & 80 & 73 & 64 & 63 & 43 & 50 & 2.2 & 2.4 \\
\hline & MO-ALT & 79 & 72 & 61 & 59 & 37 & 47 & 2.1 & 2.3 \\
\hline & SeqR & 74 & 65 & 46 & 36 & 21 & 14 & 1.7 & 1.5 \\
\hline & SRF & 62 & 60 & 40 & 36 & 17 & 13 & 1.4 & 1.4 \\
\hline & LRF & 79 & 77 & 53 & 46 & 23 & 21 & 1.8 & 1.8 \\
\hline & ILP & 58 & 52 & 24 & 22 & 2 & 4 & 1.0 & 1.0 \\
\hline \multirow{6}{*}{$\underbrace{\stackrel{8}{0}}_{\hat{\theta}}$} & MO-FIX & 80.5 & 80.7 & 65.9 & 65.2 & 44.1 & 54.3 & 2.6 & 2.8 \\
\hline & MO-ALT & 77.1 & 78.4 & 61.3 & 63 & 38.5 & 46.7 & 2.4 & 2.6 \\
\hline & SeqR & 61.9 & 60.5 & 33.5 & 31.1 & 11.1 & 5.9 & 1.4 & 1.4 \\
\hline & SRF & 68.7 & 68.5 & 38.8 & 35.2 & 10.1 & 4.1 & 1.6 & 1.5 \\
\hline & LRF & 58.9 & 56.9 & 28.5 & 24 & 6.7 & 4.3 & 1.3 & 1.2 \\
\hline & ILP & 54 & 54.3 & 19.1 & 15.2 & 1.2 & 1.7 & 1.0 & 1.0 \\
\hline
\end{tabular}

The table presents the session blocking probability (SBP) under full blocking (FB) policy and the destination blocking probability (DBP) under partial blocking (PB) policy. As it is shown, the two static strategies (MO-FIX and MO-ALT) suffer from high SBP as well as high DBP compared to the adaptive ones. Among the adaptive heuristics, SRF achieves the lowest ratio (1.4, on average) compared to the optimal solution on SBP, but suffers from a relatively high DBP (1.55, on average). In contrast, $L R F$ heuristic obtains the lowest ratio (1.25, on average) compared to the optimal solution on DBP, but with a quite high $\operatorname{SBP}$ (1.8, on average). SeqR performs more steadily, providing a good tradeoff between the other two.

\section{B. Comparison of heuristics}

1) Simulation settings: In this part, we use the realistic US Longhaul network (28 nodes, 45 links) (cf. Fig. 4(b)) as the 


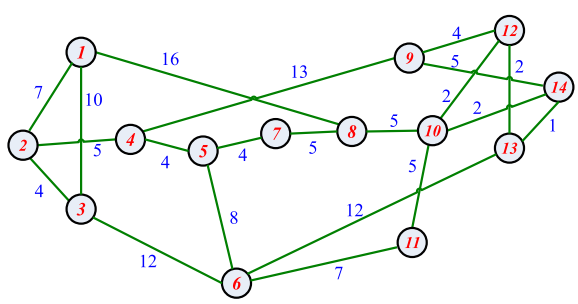

(a) NSF network topology

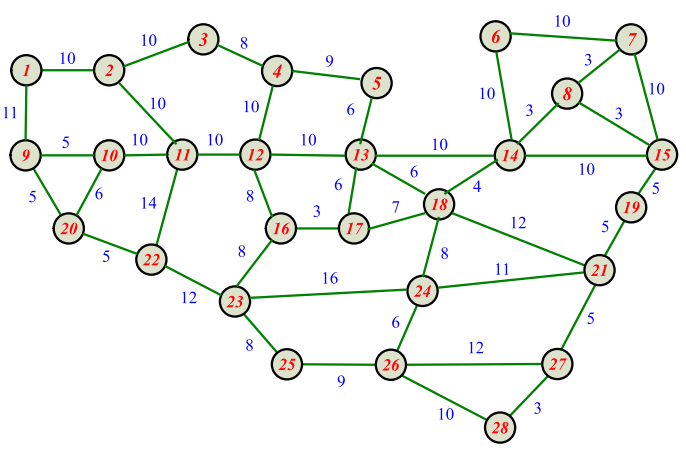

(b) US Longhaul network topology

Fig. 4: Testbeds for simulations

platform for our simulations. We conduct the simulations by setting up three contexts:

1) Given a number of requests $|R|$, the number of $\mathrm{MC}$ OXCs $S$, evaluate the solutions versus the number of wavelengths $|W|$. In particular, $|R|=200, S=6,|W|$ varies in the list $(10,20, . ., 100)$ (cf. Fig. 5).

2) Given $|R|$ requests, $|W|$ wavelengths, evaluate the solutions versus $S$ (the sparse splitting level). In particular, $|R|=200,|W|=50, S$ varies in the list $(0,3, . ., 21)$ (cf. Fig. 6).

3) Given $|W|$ wavelengths, $S$ MC-OXCs, evaluate the solutions versus $|R|$. In particular, $|W|=50, S=6$, $|R|$ varies in the list $(50,150, . ., 300)$ (cf. Fig. 7).

In our simulations: MC-OXCs are randomly selected throughout the network such that only nodes with degree larger than two are possibly selected for $\mathrm{MC}-\mathrm{OXCs}^{2}$; multicast requests are randomly generated such that their group sizes are randomly selected in the range of $(1,|V|-1)$; and 100 simulations are conducted for a given triplet $(|W|, S,|R|)$. Hence, the value of each point in the graphs is the average of 100 samples. Both session blocking probability (SBP) and destination blocking probability (DBP) are calculated to evaluate the solutions under each blocking probability policy. For the reason mentioned in Section II, we just show SBP under full blocking probability (FB) policy and DBP under partial blocking probability (PB) policy.

2) Adaptive Strategies versus Static Strategies: Since all the described adaptive strategies compute light-hierarchies for their solutions. To be fair, the two approaches should be compared on the same light-structure. To this end, we make a little modification in the $A R L H$ algorithm to compute light-trees.

\footnotetext{
${ }^{2}$ In 28 node-Longhaul topology, there are 7 nodes with degree of 2, that is why we set up to 21 nodes to be MC-OXCs.
}

The modified version is basically the same ARLH, except that whenever a destination is added, all the intermediate MI-OXCs and their adjacent arcs are removed from the wavelength graph (to retain the tree structure). In particular, we develop a lighttree version based on Sequential Request algorithm and call it Sequential Request with Light-trees (seqR-LT).

Let us focus on seqR-LT and the two static algorithms (namely, $M O-F I X$ and $M O-A L T$ ). It is easy to realize the dominance of $\operatorname{seqR}-L T$ to the other two in all the simulation contexts shown in the figures. In particular, as shown in Fig. 5(a) on SBP, the gap between seqR-LT and the two achieves maximum in both SBP and DBP at $|W|=70$ wavelengths: $33 \%$ improvement compared with $M O-F I X$, and $25 \%$ improvement compared with $M O-A L T$. Similarly, as shown in Fig. 5(b) on DBP, also at $|W|=70$ wavelengths, seqR-LT blocks $42 \%$ fewer destinations than MO-FIX, and blocks $29 \%$ fewer destinations than $M O-A L T$.

We also analyse the performances of considered strategies versus sparse splitting level of the networks (e.g., the number of MC-OXCs). As it is shown in Fig. 6, all the algorithms get lower blocking probability with denser MC-OXCs. Comparing seqR-LT with static strategies, the average gap on SBP is $23 \%$ and $17 \%$ compared with $M O-F I X$ and $M O-A L T$, respectively (cf. Fig. 6(a)); the average gap on DBP is $36 \%$ and $26 \%$, respectively (cf. Fig. 6(b)).

The results presented above is reasonable, because adaptive strategies try to route as many requests as possible by making use of the available wavelengths on the layered graph, resulting in lower blocking probability. Meanwhile, fixed strategies just compute light-trees in the physical topology neglecting the current state of network, giving high blocked probability. Fixed-alternate strategy has more choices compared with fixed strategy, it still suffers from high blocking probability due to the inherent shortcoming of static routing.

3) Light-hierarchy versus Light-tree: To show the difference between the light-hierarchy solution and the light-tree solution, we compare the performance of seqR (for lighthierarchy solution) and seqR-LT (for light-tree solution). As we can see from the figures, seqR outperforms seqR-LT in both metrics used in all the studied contexts. The advantage of seqR can be seen most clearly in Fig. 6 at very sparse splitting level. Specifically, the performance gap between the two algorithms gets pick at zero MC-OXCs: as shown in Figs. 6(a) and 6(b), seqR achieves $5.5 \%$ on SBP and $4.6 \%$ on DBP improvement over seqR-LT. On average, seqR achieves $2.8 \%$ on SBP and $2.4 \%$ on DBP better than its counterpart seqR-LT.

In short, the light-hierarchy based solutions are better than the light-tree counterparts in both blocking metrics, especially at very sparse splitting level. This is because light-hierarchies allow to exploit all the available wavelengths in current network state by taking advantage of using all possible directions (arcs) on every link and cross connects in MI-OCXs. Since there are more selections to route the requests, light-hierarchies allow to reduce blocking probability better than light-trees.

4) Comparison of adaptive light-hierarchy based strategies: As we can see from all the overall results, among three proposed adaptive strategies based on light-hierarchy, $S R F$ does best on SBP but worst on DBP, $L R F$ works best on DBP 
but worst on SBP, and seqR ranks at the middle between the other two in both metrics.

The results are predictable. Indeed, since it is easier for small requests to be totally accepted, giving high priority to them probably results in lower SBP. However, when most of small requests has been adopted, the availability of wavelengths become exhausted and the incoming (larger) requests will be blocked, causing high DBP. In contrast, provisioning the larger requests first would allow more destinations to be served, or lower DBP, however larger requests often need much of the network resources, in turn, the network resources rapidly become over-utilized and reject the incoming requests (including many small requests), causing high SBP.

In short, giving higher priority on smaller or larger requests produces own gain and loss. Choosing which one depends on the the types and objectives of applications. The applications which require the presences of all the attendants should be suitable with $S R F$ to get higher probability to take place. On the other hand, the less-restrictive applications should use $L R F$ to maximize the receivers to fulfil their communication objective. Moreover, $\operatorname{seq} R$ is a pretty choice, because it provides a tradeoff solution between the other two. Besides, seqR does not need a global information about the considered requests, it can be applied for the case of dynamic traffic where the incoming requests are unknown until they arrive.

\section{CONCLUSION AND FUTURE WORK}

This paper investigated the problem of provisioning multiple static multicast requests in sparse splitting WDM networks (MCRWA-SS). We aim at minimizing the blocking probability considering two models: full blocking probability and partial blocking probability. To this end, we employed the layered graph model and used light-hierarchies to design several adaptive algorithms to solve the problem. The contributions of this study include:

1) Provided an ILP formulation with two variants using light-hierarchies to find the exact solution for the MCRWA-SS problem.

2) Proposed three adaptive heuristic algorithms to compute the approximated solutions, in which: Smallest Request First works best under FB policy, Largest Request First achieves close optimal solution under PB policy, and Sequential Request can be used for the case of dynamic traffic (i.e., the dynamic MCRWA).

3) Showed that under sparse splitting configurations, lighthierarchy is better than light-tree for the MCRWA-SS problem in terms of blocking probability.

Note that this study supposes that all the considered requests are treated the same, meaning that the precedences of the requests are not taken into account. In some network domains, the requests are categorized into different classes according to their precedences to support various types of applications, users and specific business requirements. If it is taken into account, the proposed algorithms should be modified in such a way that the more important requests should be served first. The ILPs are also able to adapt this property by setting higher importances to the requests with higher precedences.
Moreover, many recent issues accompanied with MCRWA, including dynamic traffic, traffic grooming, QoS-aware RWA, power-aware RWA, etc., are not investigated in this work. Especially, the recent advances on multicast-related resource allocation in flexible-grid elastic optical networks (EONs) have attracted intensive research interests recently, and been treated as a potential replacement of the fixed-grid WDM networks [26], [27]. They are of our main concerns in our next study.

\section{ACKNOWLEDGMENT}

This work is realized at the LIRMM of University Montpellier 2, France. It is supported by the Vietnamese International Education Development (VIED), Vietnam; the internal grant of the Computer Science Lab (LIA), University of Avignon, France; and the open project (2013GZKF031309) of the State Key Laboratory of Advanced Optical Communication Systems and Networks, Shanghai Jiao Tong University, China.

\section{REFERENCES}

[1] Cisco, "Cisco Visual Networking Index: Forecast and Methodology, 2012-2017," in White Paper, Cisco, Tech. Rep., May 2013.

[2] X. Zhang, J. Wei, and C. Qiao, "Constrained multicast routing in WDM networks with sparse light splitting," IEEE/OSA Journal of Lightwave Technology, vol. 18, pp. 1917-1927, 2000

[3] M. Ali and J. S. Deogun, "Allocation of splitting nodes in alloptical wavelength-routed networks," Photonic Network Communications, vol. 2, pp. 247-265, 2000.

[4] D. Banerjee and B. Mukherjee, "A practical approach for routing and wavelength assignment in large wavelength-routed optical networks," Selected Areas in Communications, IEEE Journal on, vol. 14, no. 5, pp. 903-908, 1996.

[5] F. Zhou, M. Molnár, and B. Cousin, "Light-Hierarchy: The Optimal Structure for Multicast Routing in WDM Mesh Networks," IEEE Symposium on Computers and Communications(ISCC'10), vol. 15, pp. 1-6, 2010.

[6] N. Skorin-Kapov, "Routing and wavelength assignment in optical networks using bin packing based algorithms," European Journal of Operational Research, vol. 177, no. 2, pp. 1167-1179, 2007.

[7] I. Chlamtac, A. Ganz, and G. Karmi, "Lightpath communications: An approach to high bandwidth optical WAN's," Communications, IEEE Transactions on, vol. 40, no. 7, pp. 1171-1182, 1992.

[8] G. Sahin and M. Azizoglu, "Routing and wavelength assignment in all-optical networks with multicast traffic," European Transactions on Telecommunications, vol. 11, pp. 55-62, 2000.

[9] J. He, S.-H. Chan, and D. Tsang, "Routing and Wavelength Assignment for WDM Multicast Networks," IEEE GLOBECOM'01, vol. 3, pp. 1536-1540, 2001.

[10] A. Hamad, T. Wu, A. E. Kamal, and A. K. Somani, "On multicasting in wavelength-routing mesh networks," Computer Networks, vol. 50, no. 16 , pp. 3105-3164, 2006.

[11] C. Chen and S. Banerjee, "A new model for optimal routing and wavelength assignment in wavelength division multiplexed optical networks," in INFOCOM '96. Fifteenth Annual Joint Conference of the IEEE Computer Societies. Networking the Next Generation. Proceedings IEEE, vol. 1, pp. 164-171, March 1996.

[12] M. S. Kumar and P. S. Kumar, "Static lightpath establishment in WDM networks - new ILP formulations and heuristic algorithms," Computer Communications, vol. 25, no. 1, pp. 109 - 114, 2002.

[13] G.-S. Poo and A. Ding, "Layered-routing approach for solving multicast routing and wavelength assignment problem," Photonic Network Communications, vol. 13, no. 2, pp. 123-137, 2007.

[14] H. Zang, J. P. Jue, and B. Mukherjee, "A review of routing and wavelength assignment approaches for wavelength-routed optical WDM networks," Optical Networks Magazine, vol. 1, pp. 47-60, 2000.

[15] G. Markovic and V. Acimovic-Raspopovic, "An adaptive multi-criteria routing algorithm for wavelength routed optical networks," in Computer as a Tool, 2005. EUROCON 2005. The International Conference on, vol. 2, pp. 1353-1356, IEEE, 2005. 


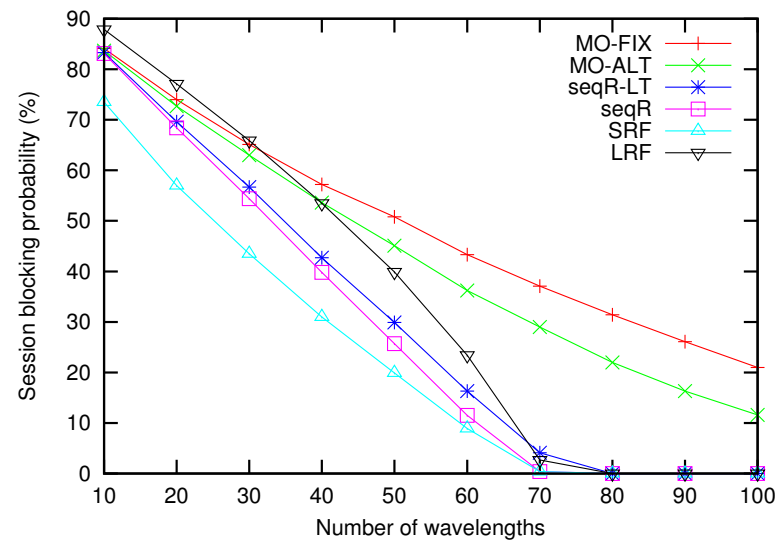

(a)

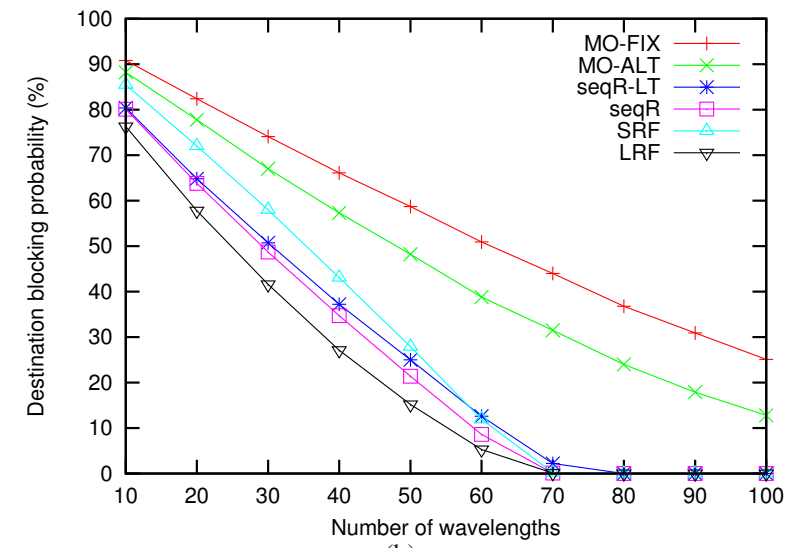

(b)

Fig. 5: Performances of the strategies on US Longhaul network with $S=6$ random MC-OXCs, $|R|=200$ requests:

a) SBP vs. Number of wavelengths; b) DBP vs. Number of wavelengths

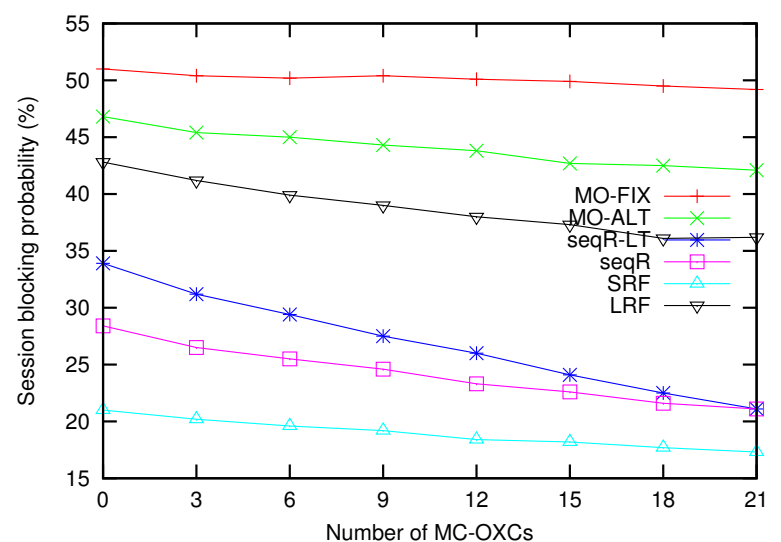

(a)

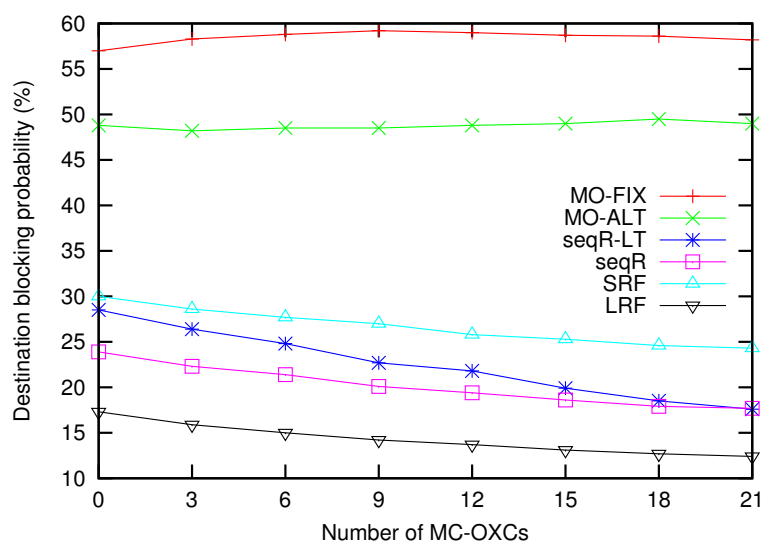

(b)

Fig. 6: Performances of the strategies on US Longhaul network with $|R|=200$ requests, $|W|=50$ wavelengths:

a) SBP vs. Number of MC-OXCs; b) DBP vs. Number of MC-OXCs

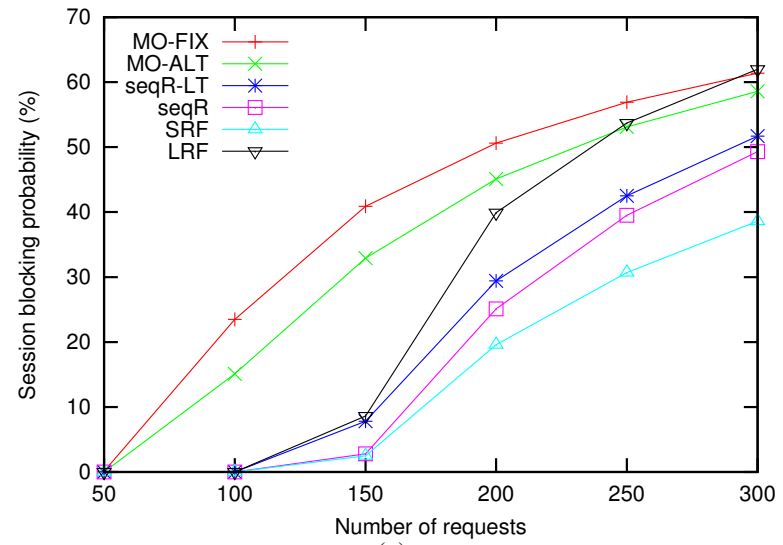

(a)

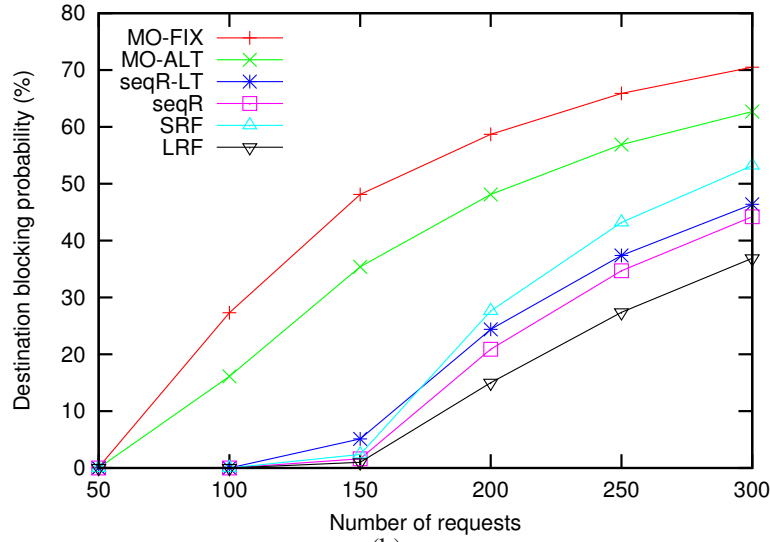

(b)

Fig. 7: Performances of the strategies on US Longhaul network with $S=6$ MC-OXCs, $|W|=50$ wavelengths: a) SBP vs. Number of requests; b) DBP vs. Number of requests

[16] X. Chu and B. Li, "Dynamic routing and wavelength assignment in the presence of wavelength conversion for all-optical networks," IEEE/ACM Transactions on Networking (TON), vol. 13, no. 3, pp. 704-715, 2005.

[17] K. Roy, M. K. Naskar, and U. Biswas, "Adaptive dynamic wavelength routing for WDM optical networks," in Wireless and Optical Communications Networks, 2006 IFIP International Conference on, pp. 4-pp,
IEEE, 2006.

[18] P. Singh, A. K. Sharma, and S. Rani, "Routing and wavelength assignment strategies in optical networks," Optical Fiber Technology, vol. 13, no. 3, pp. 191 - 197, 2007.

[19] W. Huang, L. Tang, M. Razo, A. Sivasankaran, M. Tacca, and A. Fumagalli, "Routing and wavelength assignment computed jointly for a 
given set of multicast trees reduces the total wavelength conversion," in Transparent Optical Networks (ICTON), 2010 12th International Conference on, pp. 1-5, June 2010.

[20] R. Randhawa and J. Sohal, "Static and dynamic routing and wavelength assignment algorithms for future transport networks," Optik - International Journal for Light and Electron Optics, vol. 121, no. 8, pp. $702-$ 710,2010

[21] C.-C. Hsu, H.-J. Cho, and S.-C. Fang, "Routing and wavelength assignment in optical networks from maximum edge-disjoint paths," in Genetic and Evolutionary Computing, pp. 95-103, Springer, 2014.

[22] S. Yan, J. S. Deogun, and M. Ali, "Routing in sparse splitting optical networks with multicast traffic," Computer Networks, vol. 41, no. 1, pp. 89-113, 2003.

[23] X. Wang, S. Wang, and L. Li, "A novel efficient multicast routing algorithm in sparse splitting optical networks," Photon Netw Commun, vol. 14, pp. 287-295, July 2007.

[24] C.-Y. Hsieh and W. Liao, "All-Optical Multicast Routing in Sparse Splitting WDM networks," Selected Areas in Communications, IEEE Journal on, vol. 25, pp. 51-62, August 2007.

[25] D. Danh Le, M. Molnar, and J. Palaysi, "An improved multicast routing algorithm in sparse splitting WDM networks," in International Conference on Computing, Management and Telecommunications (ComManTel'13), pp. 99-104, Jan 2013.

[26] Q. Wang and L. K. Chen, "Performance analysis of multicast traffic over spectrum elastic optical networks," in Optical Fiber Communication Conference, p. OTh3B.7, Optical Society of America, 2012.

[27] L. Gong, X. Zhou, X. Liu, W. Zhao, W. Lu, and Z. Zhu, "Efficient resource allocation for all-optical multicasting over spectrum-sliced elastic optical networks," Optical Communications and Networking, IEEE/OSA Journal of, vol. 5, pp. 836-847, Aug 2013.

[28] W. Hu and Q. Zeng, "Multicast optical cross connects employing splitterand-delivery switch," IEEE Photon. Technol. Lett., vol. 10, pp. 970-972, 1998.

[29] M. Ali and J. S. Deogun, "Cost-effective implementation of multicasting in wavelength-routed networks," EEE/OSA Journal of Lightwave Technology, vol. 18, pp. 1628-1638, 2000.

[30] M. Molnar, "Hierarchies to solve constrained connected spanning problems," tech. rep., LIRMM, University Montpellier 2, France, http://hallirmm.ccsd.cnrs.fr/lirmm-00619806, Sept. 2011.

[31] H. Takahashi and A. Matsuyama, "An approximate solution for the Steiner problem in graphs," Mathematica Japonica, vol. 24, pp. 573577, 1980.

[32] M. L. Fredman and R. Tarjan, "Fibonacci heaps and their uses in improved network optimization algorithms," in Foundations of Computer Science, 1984. 25th Annual Symposium on, pp. 338-346, Oct 1984.

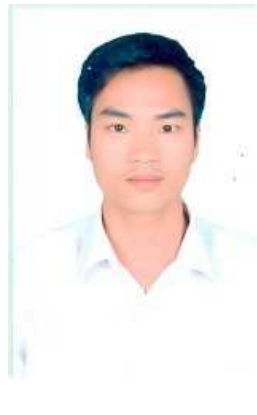

Dinh Danh Le is now a Ph.D student in Computer Science Department at laboratory LIRMM, University Montpellier 2, France. He was graduated at the Information Technology Faculty of Hong Duc University, Vietnam in 2004 and received a master degree in Computer Science at Information Technology Faculty of Vietnam National University, Hanoi in 2007. His main research topics and interests include network evaluation, multicast routing, optical networks, and combinatorial optimization.

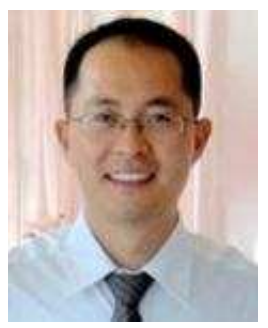

Fen Zhou is an Associate Professor at the computer science lab LIA of the University of Avignon, France. He obtained the Ph.D. degree from INSA Rennes in 2010. His research interests include combinatorial optimization for routing and resource allocation in optical networks, content delivery networks $(\mathrm{CDN})$ and vehicular ad-hoc networks (VANETs)

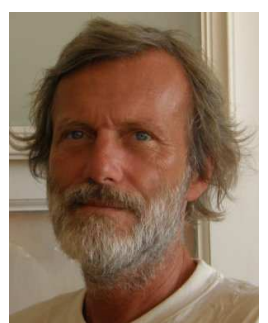

Miklós Molnár is with the University Montpellier 2, laboratory LIRMM and he is a full professor in Computer Science. He was graduated at the Faculty of Electrical Engineering, University BME of Budapest in 1976 and received the Ph.D. degree in Computer Science from the University of Rennes 1 in 1992 and the French HDR degree in 2008 His main research activity is in combinatorial optimization to solve network related problems. His results are related to NP-hard optimization problems, constrained spanning and Steiner problems, routing algorithms for unicast, in-cast and multicast communications in optical and in wireless networks, dependable communications, energy aware protocols and optimization. 